\title{
EFECTO DEL DETERGENTE DOMÉSTICO ALQUIL ARIL SULFONATO DE SODIO LINEAL (LAS) SOBRE LA MORTALIDAD DE TRES CARACOLES DULCEACUÍCOLAS EN EL PERÚ
}

\author{
José Iannacone ${ }^{1}$ y Lorena Alvariño
}

\section{Resumen}

Se realizó una evaluación ecotoxicológica estandarizada del surfactante aniónico alquil aril sulfonato de sodio lineal (LAS), en la Universidad Federico Villarreal, Lima, Perú, empleando a tres caracoles dulceacuícolas Melanoides tuberculata (Müller, 1774) (Thiaridae), Physa venustula (Gould, 1847) (Physidae) y Heleobia cumingii (Orbigny, 1835) (Hydrobiidae) como herramientas para la evaluación de riesgos ambientales. La colecta de las tres especies de caracoles se realizó en diversos humedales naturales y artificiales del departamento de Lima, Perú. M. tuberculata fue colectada de la Laguna de la Molina $\left(15^{\circ} 5^{\prime} \mathrm{LS}, 75^{\circ} 57^{\prime} \mathrm{LO}\right), P$. venustula de las lagunas de Villa $\left(12^{\circ} 13^{\prime} \mathrm{LS}, 77^{\circ} 01^{\prime} \mathrm{LO}\right)$ y $H$. cumingii de las lagunas de Puerto Viejo (12 $\left.35,2^{\prime} \mathrm{LS}, 76^{\circ} 42,2^{\prime} \mathrm{LO}\right)$. Los tres moluscos fueron individualizados y criados en acuarios de vidrio de $30 \mathrm{~cm}$ x $20 \mathrm{~cm} 20 \mathrm{~cm}$ y se aclimataron en condiciones estandarizadas de laboratorio por una semana previa a los bioensayos, empleando agua filtrada a $0,54 \mu$ de abertura procedentes de las lagunas de colecta y agua de grifo hervida fría $(1: 1 \mathrm{v} / \mathrm{v})$. Los gasterópodos fueron alimentados adlibitum empleando el alga verde filamentosa Cladophora glomerata y la microalga Chlorella vulgaris Beij. Los valores determinados de la concentración letal media $\left(\mathrm{CL}_{50}\right)$ para el efecto letal fueron a $48 \mathrm{~h}$ de exposición respectivamente: $M$. tuberculata $201,07 \mathrm{mg} \mathrm{L}^{-1} ; P$. venustula $71,41 \mathrm{mg} \mathrm{L}^{-1}$ y $H$. cumingii $82,93 \mathrm{mg} \mathrm{L}^{-1}$. Además no existieron diferencias en la toxicidad sobre los caracoles de tres formulaciones comerciales de estos detergentes domésticos. Se proponen a estas tres especies de moluscos como herramientas para la evaluación de riesgos ambientales por detergentes domésticos.

Palabras claves: caracoles dulceacuícolas, detergentes domésticos, Heleobia, LAS, Melanoides, Physa, surfactante.

A standardized ecotoxicological evaluation of anionic surfactant linear alkyl aryl sulfonate (LAS) was realized at Federico Villarreal University, Lima, Peru, employing three freshwater snails Melanoides tuberculata (Müller, 1774) (Thiaridae), Physa venustula (Gould, 1847) (Physidae) and Heleobia cumingii (Orbigny, 1835) (Hydrobiidae) as tools for environmental risk assessment. The collection of three species of snails was done in diverse natural and artificial wetlands in the departament of Lima, Peru. M. tuberculata in "La Molina" lagoon (15 $5^{\circ}$ 'LS, $\left.75^{\circ} 57^{\prime} \mathrm{LW}\right), P$. venustula in Villa lagoon $\left(12^{\circ} 13^{\prime} \mathrm{LS}, 77^{\circ} 01^{\prime} \mathrm{LW}\right)$ and also $H$. cumingii in Puerto Viejo lagoon $\left(12^{\circ} 35,2^{\prime} \mathrm{LS}, 76^{\circ} 42,2^{\prime} \mathrm{LW}\right)$. The three molluscs were individualized and reared in glass aquariums of $30 \mathrm{~cm} \times 20 \mathrm{~cm} 20 \mathrm{~cm}$ and were acclimated in standardized laboratory conditions a week before the bioassay, employing water filtered at $0.54 \mathrm{u}$ of mesh proceeding from the collection lagoons and cold boiled potable water $(1: 1 \mathrm{v} / \mathrm{v})$. The gastropods were feed $a d$ libitum employing filamentous green algae Cladophora glomerata and the microalgae Chlorella vulgaris Beij. The values determined for mean lethal concentration $\left(\mathrm{LC}_{50}\right)$ for lethal effect of this surfactant were at $48 \mathrm{~h}$ of exposure respectively: M. tuberculata $201.07 \mathrm{mg} \mathrm{L}^{-1}$; P. venustula 71.41 $\mathrm{mg} \mathrm{L}^{-1}$ and $H$. cumingii $82.93 \mathrm{mg} \mathrm{L}^{-1}$. We did not find differences in the toxicity on freshwater snails by three commercial formulations of these household detergents. An ecotoxicological assay using these three species of molluscs is proposed as a tool for the evaluation of environmental risks of household detergents.

Keywords: freshwater snails, Heleobia, household detergents, LAS, Melanoides, Physa, surfactant.

\section{Introducción}

La contaminación dulceacuícola ocasionada por las actividades antropogénicas constituye uno de los problemas de mayor trascendencia en nuestros tiempos. Los detergentes domésticos, se encuentran entre los contaminantes de naturaleza orgánica de mayor trascendencia al nivel mundial (Lewis, 1986; Alvarez et al., 1999). Romero (1996) señala que los detergentes causan problemas de espuma en aguas superficiales, lagos, plantas de aguas residuales, y en general en sitios de mezcla turbulenta de aguas residuales (Ying et al., 2002). La biodegradabilidad

\footnotetext{
${ }^{1}$ Laboratorio de Ecofisiología. Facultad de Ciencias Naturales. Universidad Nacional Federico Villarreal. Email: joseiannacone@hotmail.com
} 
de los detergentes domésticos es muy variable (Jensen, 1999; Temara et al., 2001). Dependiendo de su estructura química, pueden ser fácilmente descompuestos o difíciles de utilizar por las bacterias. Los fabricados con base en ABS (Alquil benceno sulfonato de sodio ramificado) son resistentes al ataque biológico por su composición molecular ramificada y por la adhesión de los anillos bencénicos a los átomos terciarios de carbono de los grupos de cadena ramificada. Los fabricados con base en LAS (Alquil benceno sulfonato de sodio lineal) son biodegradables en condiciones aeróbicas, pero resistentes a la actividad bacteriana anaeróbica. En América y Europa, desde fines de los sesentas los detergentes del tipo ABS fueron reemplazados con detergentes del tipo LAS (Kimerie 1989; Argese et al., 1994; Eichhorn et al., 2001).

La evaluación del riesgo ambiental es un proceso de asignación de magnitudes, rangos y probabilidades a los efectos adversos que pueden derivar del uso de sustancias químicas (Wong \& Dixon, 1995). Los riesgos ecológicos por lo general son juzgados basándose en el efecto sobre los organismos o la comunidad de poblaciones y en los valores finales, como la concentración letal media $\left(\mathrm{CL}_{50}\right)$, calculados a partir de ensayos ecotoxicológicos (Iannacone \& Alvariño, 2002; Iannacone et al., 2002a). Los ensayos de toxicidad son modificados por variables como factores físicos y químicos, tiempo de exposición, agente químico y disponibilidad (Lam, 1996). Iannacone et al. (1998) demostraron que muchas especies son útiles para evaluar la ecotoxicidad del agua, suelo, afluentes y sedimentos; entre ellos las bacterias, algas, plantas acuáticas, crustáceos, insectos, moluscos, peces, etc.

Los organismos acuáticos como los moluscos tienen una función trófica de importancia en la dinámica de los ecosistemas acuáticos, además son herramientas biológicas esenciales para evaluar la respuesta a contaminantes (Iannacone et al., 2001).

Algunos moluscos dulceacuícolas como Pomacea canaliculata (Lamarck) son utilizados en la dieta humana (Iannacone et al., 2002b). Otros, están involucrados en la transmisión de diferentes zoonosis (esquistosomiasis, fasciolasis, paragonimiasis, angiostrongiliasis $\mathrm{y}$ otras), importantes en salud pública y veterinaria en la región neotropical. Además, existen especies que son efectivos controladores biológicos de gastrópodos hospederos intermediarios de las parasitosis mencionadas (Paredes et al., 1998).

El caracol dulceacuícola prosobranchia Melanoides tuberculata (Müller, 1774) (Thiaridae) es originario de Malasia, por lo cual son llamados "Caracoles trompeta de Malasia". Son dioicos, y existe dimorfismo sexual caracterizado por el mayor tamaño del macho. Se reproducen rápidamente y son vivíparos. Su hábitat son las lagunas fangosas y pedregosas debido a que prefieren ocultarse bajo estos sustratos durante el día y emerger durante la noche para alimentarse (Perera et al., 1987). Se alimentan de microalgas y plantas, y en algunos casos prefieren el detritus. Además pueden resistir altos valores de alcalinidad del agua (Ferrer, 1993-1994).

En el Perú se encuentra el pulmonado Physa venustula (Gould, 1847) (Physidae) la cual se escogió como organismo prueba por ser una especie indicadora de la calidad del agua y por su amplia distribución (Iannacone y Alvariño, 1999). $P$. venustula es un gasterópodo propio de la región Neotropical (Perú, Ecuador y Colombia), muy bien representado en lagos, estanques y aguas tranquilas de ríos. En Perú, se encuentra especialmente en diferentes ambientes costeros, principalmente en el ambiente natural de los Pantanos de Villa, Lima, Perú, y además se ha encontrado en ambientes artificiales como las lagunas de oxidación (Vivar et al., 1998). Se les puede encontrar principalmente asociados a Hydrocotyle, Myriophyllum, Azolla, algas filamentosas y en las paredes de acequias. Habita en agua limpia con abundante vegetación, puede desplazarse en suelo limoso y vivir en ambientes con un $\mathrm{pH}$ entre 6,4 y 7,2. Sin embargo, los datos de la alimentación y condiciones físico-químicas del hábitat de $P$. venustula son escasos (Vivar et al., 1998).

Heleobia cumingii (Orbigny, 1835) (Hydrobiidae) se encuentra distribuido en el Perú, Ecuador y Colombia. En el Perú se le encuentra en los departamentos de Lambayeque, La Libertad, Lima e Ica, en diversos ambientes lénticos y lóticos. En los Pantanos de Villa sólo se encuentra a este prosobranchia en las acequias laterales que llegan a la laguna principal (Vivar et al., 1996). Esta especie es capaz de soportar $\mathrm{pH}$ variable de 6,4 a 8,9 . Se le encuentra asociada principalmente en las raíces de Eichhornia, Myriophyllum, Hydrocotyle y Pistia (Vivar et al., 1998).

El objetivo principal del trabajo fue determinar la concentración letal media $\left(\mathrm{CL}_{50}\right)$ del surfactante aniónico alquil aril sulfonato de sodio lineal (LAS) en tres formulaciones comerciales, empleandose tres caracoles dulceacuícolas M. tuberculata (Thiaridae), $P$. venustula (Physidae) y H. cumingii (Hydrobiidae) como herramientas para ensayos de ecotoxicidad para la evaluación de riesgos ambientales por detergentes domésticos.

\section{Materiales y métodos}

Caracoles dulceacuícolas

Los adultos de $M$. tuberculata se colectaron con la ayuda de un cucharón de las orillas arenosas de los humedales de las Lagunas de la Molina (15 ${ }^{\circ}{ }^{\prime} \mathrm{LS} ; 7^{\circ}$ 57' LO), Lima, Perú. Los caracoles en presencia de la luz del día se encontraron refugiados debajo del sustrato arenoso-fangoso. Los adultos de $P$. venustula se colectaron con la ayuda de un cucharón de las orillas de los humedales de las Lagunas de Villa $\left(12^{\circ}\right.$ 
13' LS, $77^{\circ} 01^{\prime}$ LO), Lima, Perú. Los adultos de $H$. cumingii se colectaron con la ayuda de un cucharón de las orillas de los humedales de las Lagunas de Puerto Viejo ( $12^{\circ} 35,2^{\prime}$ LS, $76^{\circ} 42,2^{\prime}$ LO), Lima, Perú. Posteriormente los caracoles fueron trasladados al laboratorio en recipientes de plástico de 2000 a 4000 $\mathrm{mL}$ de capacidad, con sustrato en el fondo. Los caracoles fueron criados en acuarios de vidrio de 30 $\mathrm{cm} \times 20 \mathrm{~cm} \times 20 \mathrm{~cm}$ de capacidad y aclimatados por siete días previos a los bioensayos empleando agua filtrada a $0,54 \mu$ de abertura procedentes de las lagunas de colecta y agua de grifo hervida fría (1:1 $\mathrm{v} / \mathrm{v}$ ). Los caracoles M. tuberculata fueron alimentados con el alga verde Cladophora glomerata colectadas de la misma laguna. La longitud total promedio de M. tuberculata empleados en los bioensayos fue de 7,56 $\mathrm{mm} \pm 1,27 \mathrm{~mm}(\mathrm{CV}=16,79 \%)$. P. venustula y $H$. cumingii fueron alimentados con la microalga verde Chlorella vulgaris Beij. La longitud total promedio de $P$. venustula empleados en los bioensayos fue de 4,86 $\mathrm{mm} \pm 0,95 \mathrm{~mm}(\mathrm{CV}=19,54 \%)$. La longitud total promedio de $H$. cumingii empleados en los bioensayos fue de $3,10 \mathrm{~mm} \pm 0,11 \mathrm{~mm}(\mathrm{CV}=3,54 \%)$.

Surfactante aniónico alquil aril sulfonato de sodio lineal (LAS)

Los detergentes domésticos comerciales usados fueron: Ace Poder Limón $\AA$, Ariel Limón ${ }^{\circledR}$ y Magia Blanca ${ }^{\circledR}$. En todos los casos el Ingrediente Activo principal de la mezcla compleja de surfactantes homólogos, oligómeros e isómeros fue el alquil aril sulfonato de sodio $\mathrm{C}_{11.8}(90 \%)$, que corresponde a los detergentes llamados blandos o LAS. Los tres surfactantes comerciales presentan como compuestos adicionales $\mathrm{NaSO}_{4}$ y $\mathrm{NaPO}_{4}$. Solo Magia blanca ${ }^{\circledR}$ carece de aditivos biológicos (limón) y de agentes dispersantes. Se preparó una solución madre al 0,09 $\%$ con $1 \mathrm{~g}$ del producto de cada detergente separadamente en $1 \mathrm{~L}$ de agua destilada $\left(450 \mathrm{mg} \mathrm{IA} \mathrm{L}^{-}\right.$ ${ }^{1}$ ). A partir de estas soluciones se prepararon las diferentes concentraciones para los ensayos ecotoxicógicos.

Para $M$. tuberculata las cinco concentraciones fueron $225 ; 112,5 ; 56,25 ; 28,12$ y $14,06 \mathrm{mg} \mathrm{L}^{-1}$, mientras que para $P$. venustula y $H$. cumingii fueron $450 ; 225 ; 112,5 ; 56,25$ y $28,12 \mathrm{mg} \mathrm{L}^{-1}$. El agua de dilución usada para preparar las concentraciones fueron agua hervida fría y filtrada a $0,54 \mu$ (Iannacone et al., 2000). La solución madre y el agua de dilución fueron ajustadas a $\mathrm{pH}$ siete con $\mathrm{HCl} 1 \mathrm{M} \mathrm{o} \mathrm{H}_{2} \mathrm{SO}_{4} 1 \mathrm{~N}$.

\section{Parámetros físico-químicos}

El $\mathrm{pH}$ y la conductividad del agua hervida fría y filtrada fueron medidos en la solución madre y al inicio de los bioensayos. La determinación de los fosfatos se realizó mediante la técnica del ácido ascórbico (APHA, 1989) y para los sulfatos se empleó el procedimiento del sulfato de sodio anhidro $\left(\mathrm{Na}_{2} \mathrm{SO}_{4}\right)$ (ASTM, 1991).

\section{Pruebas ecotoxicológicas}

Una vez obtenidas los caracoles, se aclimataron de $M$. tuberculata, $P$. venustula y $H$. cumingii de una cohorte de tallas homogéneas y se procedió a realizar los ensayos de ecotoxicidad (Iannacone y Alvariño, 1999). Las pruebas estuvieron compuestas de un control y cinco concentraciones nominales de los detergentes; para cada prueba con $M$. tuberculata se necesitó un total de 240 especímenes y para $P$. venustula y $H$. cumingii se emplearon 120 individuos por bioensayo. En cada envase se colocaron 10 individuos de M. tuberculata y 5 para $P$. venustula y $H$. cumingii que se distribuyeron al azar en cada una de las cuatro repeticiones. Las lecturas de mortalidad se realizaron a las $48 \mathrm{~h}$ de exposición. Al inicio de cada ensayo se ajustó a un pH de 7,0 con una solución de $\mathrm{HCl} 1 \mathrm{M}$ o $\mathrm{H}_{2} \mathrm{SO}_{4} 1 \mathrm{~N}$. Las cinco concentraciones siguieron un incremento x 2. Para la discriminación de mortalidad se usó el criterio propuesto por Iannacone y Alvariño (1999). Se consideró muerto el individuo incapaz de realizar algún tipo de movimiento en la placa de recuento, como mover el pie, la concha ó los tentáculos cefálicos durante $15 \mathrm{~s}$ de observación al estereoscopio.

\section{Análisis de datos}

Las pruebas de ecotoxicidad aguda para los tres detergentes se realizaron en cuatro repeticiones con las cinco concentraciones nominales señaladas previamente y un control en un diseño en bloque completamente aleatorizado: $6 \times 4$. Los porcentajes de mortalidad se transformaron a arcoseno $\sqrt{ } \%$, con el fin de ajustar los datos a una distribución normal (Zar, 1996). Se realizó un análisis de varianza (ANDEVA) de una vía con el propósito de analizar las diferencias entre las concentraciones de detergente aplicado y entre las repeticiones. Se determinó el grado de significancia, para que en los casos necesarios, fuera contrastado con la prueba a posteriori de StudentNewman-Keuls (S-N-K) usando letras alfabéticas. El nivel de significancia fue de $\alpha=0,05$ (Zar, 1996). Todos los cálculos estadísticos se realizaron con el paquete SPSS, para Windows 95 . La $\mathrm{CL}_{50}$, sus respectivos límites de confianza al $95 \%$ se calcularon usando el programa computarizado de la EPA Probit 1,5-1983. Además se determinaron dos valores de efectos subletales: NOEC (Concentración de efectos no observables) y LOEC (Concentración más baja de efectos observables) para cada uno de los ensayos evaluados.

\section{Resultados}

La Tabla 1 muestra los valores de toxicidad aguda en términos de $\mathrm{CL}_{50}$, NOEC y LOEC para $M$. tuberculata. No se observaron diferencias 
significativas en términos de $\mathrm{CL}_{50}$ para las tres formulaciones comerciales de alquil aril sulfonato de sodio (LAS). La $\mathrm{CL}_{50}$ promedio sobre $M$. tuberculata fue de 201,07 mg IA L $\mathrm{L}^{-1}$. El ariel Limón ${ }^{\circledR}$ a la concentración de $225 \mathrm{mg} \mathrm{L}^{-1}$ presentó mortalidades diferentes a las otras dos formulaciones. La Tabla 2 y 3 muestran los valores de toxicidad aguda en términos de $\mathrm{CL}_{50}$, NOEC y LOEC para $P$. venustula y $H$. cumingii, respectivamente. Para $P$. venustula la $\mathrm{CL}_{50}$ promedio fue de $71,41 \mathrm{mg} \mathrm{IA} \mathrm{L}^{-1}$. En contraste para $H$. cumingii la $\mathrm{CL}_{50}$ promedio fue de $82,93 \mathrm{mg} \mathrm{IA} \mathrm{L}^{-1}$. No se observaron diferencias significativas en términos de $\mathrm{CL}_{50}$ para las dos formulaciones comerciales a base del alquil aril sulfonato de sodio (LAS) empleadas sobre estos dos caracoles. La secuencia de toxicidad aguda presentó el siguiente orden: $P$. venustula $>H$. cumingii $>$ M. tuberculata.

En todos los casos los análisis estadísticos mostraron que no existieron diferencias significativas entre las repeticiones, pero sí entre las concentraciones (Tablas 1 - 3).

Además, la Tabla 1 indica los valores de fosfatos y sulfatos encontrados para los tres detergentes domésticos comerciales. Se observó para fosfatos y sulfatos el siguiente orden secuencial de mayor a menor: Ace Poder limón ${ }^{\circledR}>$ Ariel limón ${ }^{\circledR}>$ Magia blanca ${ }^{\circledR}$ (Tabla 1).

La tabla 4 muestra en forma comparativa los valores de las pruebas de toxicidad para el ensayo con partículas mitocondriales, con un pez, con dos invertebrados, con dos microalgas y con los tres caracoles dulceacuícolas. Mostrando que los valores obtenidos con los caracoles son numéricamente mayores en comparación con los otros organismos biológicos.

\section{Discusión}

El empleo de estos tres caracoles bénticos dulceacuícolas pueden ser útiles para monitorear detergentes aniónicos del grupo de los LAS directamente de las aguas residuales. En cambio para cuerpos de agua dulceacuícolas o en desagües tratados es necesario una fase de preconcentración de la muestra de agua antes de realizar los bioensayos ecotoxicológicos (Argese et al., 1994).

Pettersson et al. (2000) han determinado la toxicidad aguda de 25 detergentes comerciales en base al LAS, sobre el cladócero Daphnia magna, encontrando que la toxicidad en términos de $\mathrm{CL}_{50}$ a 48 $\mathrm{h}$ de exposición varió entre 4 a $85 \mathrm{mg}$ de IA $\mathrm{L}^{-1}$. En nuestro estudio dos de los moluscos: $P$. venustula y $H$. cumingii presentaron valores coincidentes con este invertebrado (Tabla 4). La respuesta diferente y de menor sensibilidad de la $\mathrm{CL}_{50}$ a $48 \mathrm{~h}$ de exposición de M. tuberculata pudiera ser presumiblemente adscrita a diferencias en la bioquímica y fisiología de este caracol en comparación con los otros organismos biológicos (Tabla 4). Además, en un estudio de macrozoobentos de las Lagunas de Puerto Viejo, Lima, Perú, se encontró a $M$. tuberculata en la mayoría de las estaciones y muestreos, señalando su amplia valencia y plasticidad ecológica (Iannacone et al., 2002c).

A pesar de la toxicidad observada por LAS sobre estos tres caracoles (Tabla 4). La molécula del surfactante LAS, presentan una alta biodegradabilidad aeróbica, pues se empiezan a degradar con la carboxilación del grupo terminal metilo por medio de una serie de $\beta$-oxidaciones formando cadenas cortas de SPCs (Ácidos sulfofenilos) (Argese et al., 1994). Este proceso parece ser el responsable para la marcada reducción de la surfactancia, potencial de bioacumulación y toxicidad de la molécula LAS (Lewis, 1986). Además, su corto tiempo de vida medio de aproximadamente de 1 a 3 semanas, generalmente previene su acumulación en la biota, en el agua y en el suelo (Jensen, 1999). Temara et al. (2001) señalan que el riesgo de LAS a los organismos pelágicos acuáticos es bajo, debido a que la concentración máxima esperada es de 3 a 30 veces más baja que la $\mathrm{CL}_{50}$ y de la NOEC.

Finalmente recomendamos el empleo en ensayos ecotoxicológicos para la evaluación de detergentes domésticos a $P$. venustula y $H$. cumingii debido a su mayor sensibilidad observada al LAS en comparación con M. tuberculata.

\section{Referencias bibliográficas}

Álvarez G., Medina G. y Sánchez G. 1999. Efecto del detergente biodegradable (Aquil sulfonato de sodio) en el consumo de oxígeno y tasa de filtración del bivalvo Semimytilus algosus. Rev. per. Biol. 6: 68-74.

APHA. 1989. Standard methods for examination of water and wastewater. $17^{\text {th }}$ Ed. American Public Association (APHA), Washington, D.C. USA.

Argese E., Marcomini A., Miana P., Bettiol C. \& Perin G. 1994. Submitocondrial particle response to linear alkylbenzene sulfonates, nonylphenol polyethoxilates and their biodegradation derivatives. Environ. Toxicol. Chem. 13: 737-742.

ASTM. 1991. Subcommittee D19.05.1991. Annual Book of ASTM Standards. Vol 11.01.

Eichhorn P., Flavier M.E., Paje M.L. \& Kneppe T.P. 2001. Occurrence and fate of linear and branched alkylbenzenesulfonate and their metabolites in surface waters in the Philippines. Sci. Total Environ. 269: 75-85.

Ferrer J.R., Perera G., Yong M., Gutierrez A. y Sanchez J. (1993-1994). Evidencias de formas diferentes en dos poblaciones de Melanoides tuberculata en Cuba: Estudio morfométrico. Walkerana. 7: 23-28.

Iannacone J. y Alvariño L. 1999. Ecotoxicidad aguda de metales pesados empleando juveniles del 
caracol de agua dulce Physa venustula (Gould, 1847) (Mollusca). Gayana. 63: 101-110.

Iannacone J., Alvariño L. y Dale W. 1998. Pruebas ecotoxicológicas como una herramienta para la evaluación del impacto ambiental. Bol. Lima (Perú). 113: 53-68.

Iannacone J., Alvariño L. y Ramírez P. 2000. Una técnica de bioensayo empleando a los ciliados de vida libre Stentor coeruleus Enrenberg y Spirostomum ambiguum Enrenberg para la evaluación de los efectos del mercurio y arsénico. Acta Toxicol. Argent. 8: 5-9.

Iannacone J. y Alvariño L. 2002. Evaluación del riesgo ambiental del insecticida cartap en bioensayos con tres invertebrados. Agric.Téc. 62: 366-374.

Iannacone J., Caballero C. y Alvariño L. 2002a. Empleo del caracol de agua dulce Physa venustula Gould como herramienta ecotoxicológica para la evaluación de riesgos ambientales por plaguicidas. Agric. Téc. 62: 212-225.

Iannacone J., García J., Vela H., Ticona C., Torres E., Quinte G. y Vidarte K. 2001. Toxicidad y bioacumulación de plomo en Perumytilus purpuratus (Lamarck, 1819) "chorito" (Bivalvia). Bol. Soc. Quim. Perú. 67: 89-98.

Iannacone J., Pantoja C. y Malpartida C. 2002b. Efecto ecotóxico del mercurio sobre Pomacea canaliculata (Orbigny) (Mollusca: Ampullariidae), procedentes del distrito de Aucayacu, Huánuco, Perú. Libro de Resúmenes del V Congreso Peruano de Parasitología, 2 al 5 de octubre del 2002. Universidad Nacional de Trujillo, Perú.

Iannacone J., Mansilla C.J. y Ventura Z.V. 2002c. Macrozoobentos en las lagunas de Puerto Viejo, Lima-Perú. Libro de Resúmenes de la XI Reunión Científica del Instituto de Investigación de Ciencias Biológicas "Antonio Raimondi" (ICBAR), 24 - 26 Abril del 2002. p. 49.

Jensen J. 1999. Fate and effects of linear alkylbenzene sulphonate (LAS) in the terrestrial environment. Sci. Total Environ. 226: 93-111.

Kimerie, R.A. 1989. Aquatic and terrestial ecotoxicology of linear alkylbenzene sulfonate. Tenside Det. 26: 169-176.

Lam, P. 1996. Sublethal effects of cadmium on the energetics of a tropical freshwater snail, Brotia hainanensis (Brto, 1872). Environ. Toxicol. Water Qual. 11: 345-349.

Lewis, M. A. 1986. Comparison of the effects of surfactants on freshwater phytoplankton communities in experimental enclosure and on algal population growth in the laboratory. Environ. Toxicol. Chem. 5: 319-332.

Morgan E.C. \& Oude N.T. 1993. Detergents. pp. 130154. In: Handbook of Ecotoxicology. P. Calow (ed.).
Paredes C., Huamán P., Ramírez R., Vivar R., Cardoso F. y Vera V. 1998. Diversidad de los moluscos en el Perú. Acta Zoológica Mexicana. (Vol. Especial): 133-142.

Perera G., Yong M. \& Sanchez R. 1987. First record of an ecological studies on Melanoides tuberculata in Cuba. Walkerama. 2:165-171.

Pettersson A., Adamssson M. \& Dave G. Toxicity and detoxification of Swedish detergents and softener products. Chemosphere . 41: 1611-1620.

Romero R.J.A. 1996. Acuiquímica. Ed. Presencia. Santafé de Bogotá. Colombia.

Temara A., Carr G., Webb S., Versteeg D. \& Feijtel T. 2001. Marine risk assessment: linear alkylbenzenesulponates (LAS) in the North Sea. Mar. Pollut. Bull. 42: 635-642.

Vivar R., Larrea H., Huamán P., Yong M. \& Perera G. 1996. Some ecological aspects of the freshwater molluscan fauna of Pantanos de Villa, Lima, Peru. Malacol. Rev. 29: 65-68.

Vivar R., Ramírez R. y Huamán P. 1998. Moluscos de los Pantanos de Villa. Serie de divulgación UNMSM-MHN 11. Cano, A.; Young, K. (Eds.).

Wong P.T.S. \& Dixon D.G. 1995. Bioassessment of water quality. Environ. Toxicol. Water Qual. 10: 9-17.

Ying G.G., Williams B. \& Kookana R. 2002. Environmental fate of alkylphenol ethoxylates- a review. Environ. Int. 28: 215-226.

Zar J.H. 1996. Biostatistical analysis. $3^{\text {th }}$ Ed. PrenticeHall, New York, USA. 
Tabla 1. Concentración Letal media $\left(\mathrm{CL}_{50}\right)$, NOEC, LOEC de Melanoides tuberculata expuesta al detergente alquil aril sulfonato de sodio (LAS) por $48 \mathrm{~h}$.

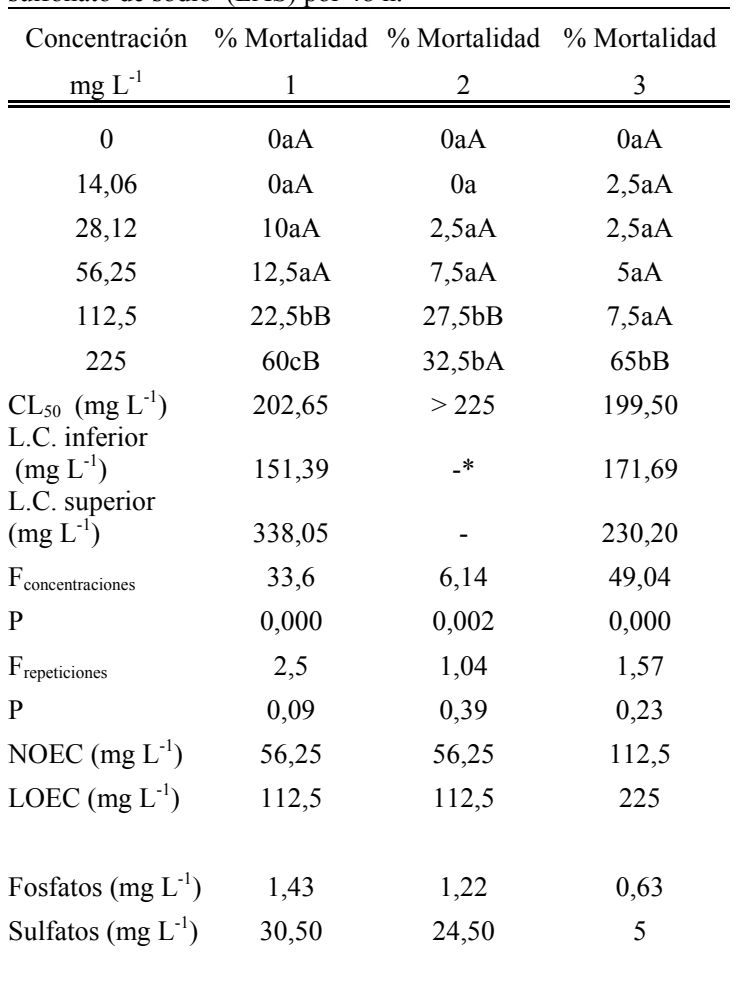

\footnotetext{
$1=$ Ace poder

limón ${ }^{\circledR}$

$2=$ Ariel

Limón $\mathbb{}$

$3=$ Magia

blanca $\mathbb{R}$

$*$ = No calculado

* = Concentración en una solución de $225 \mathrm{mg} \mathrm{L}^{-1}$ de Alquil Aril sulfonato de sodio.

L.C. = límite de confianza;

$\mathrm{F}=$ estadístico de Fisher;

$\mathrm{P}=$ Probabilidad

NOEC $=$ concentración de efectos no observables;

LOEC $=$ concentración más baja de efectos observables

Letras iguales minúsculas en una misma línea vertical indican porcentaje de mortalidad estadísticamente iguales, según los resultados de la prueba de Student-Newman-Keuls (S-N-K). (SPSS versión 7,5).

Letras iguales mayúsculas en una misma línea horizontal indican porcentaje de mortalidad estadísticamente iguales según los resultados de la prueba de Student-Newman-Keuls (S-N-K) (SPSS versión 7,5)
}

Tabla 2. Concentración Letal media $\left(\mathrm{CL}_{50}\right)$, NOEC, LOEC de Physa venustula expuesta al detergente alquil aril sulfonato de sodio (LAS) por $48 \mathrm{~h}$.

\begin{tabular}{|c|c|c|}
\hline $\begin{array}{c}\text { Concentración } \\
\mathrm{mg} \mathrm{L}\end{array}$ & $\begin{array}{c}\% \text { Mortalidad } \\
1 \\
\end{array}$ & $\begin{array}{c}\% \text { Mortalidad } \\
2 \\
\end{array}$ \\
\hline 0 & $0 \mathrm{aA}$ & $0 \mathrm{aA}$ \\
\hline 28,12 & $5 \mathrm{aA}$ & $15 \mathrm{aA}$ \\
\hline 56,25 & $25 \mathrm{abA}$ & $15 \mathrm{aA}$ \\
\hline 112,5 & $75 \mathrm{bA}$ & $90 \mathrm{bA}$ \\
\hline 225 & $100 \mathrm{cA}$ & $100 \mathrm{bA}$ \\
\hline 450 & $100 \mathrm{cA}$ & $100 \mathrm{bA}$ \\
\hline $\begin{array}{l}\mathrm{CL}_{50}\left(\mathrm{mg} \mathrm{L}^{-1}\right) \\
\text { L.C. inferior }(\mathrm{mg}\end{array}$ & 75,89 & 66,97 \\
\hline $\begin{array}{l}\left.\mathrm{L}^{-1}\right) \\
\text { L.C. superior }\end{array}$ & 61,44 & 5,24 \\
\hline$\left(\mathrm{mg} \mathrm{L}^{-1}\right)$ & 93,44 & 428,05 \\
\hline $\mathrm{F}_{\text {concentraciones }}$ & 46,02 & 67,25 \\
\hline $\mathrm{P}$ & 0,00 & 0,00 \\
\hline $\mathrm{F}_{\text {repeticiones }}$ & 2,10 & 1,44 \\
\hline $\mathrm{P}$ & 0,17 & 0,31 \\
\hline $\operatorname{NOEC}\left(\mathrm{mg} \mathrm{L}^{-1}\right)$ & 112,5 & 112,5 \\
\hline $\operatorname{LOEC}\left(\mathrm{mg} \mathrm{L}^{-1}\right)$ & 225 & 225 \\
\hline
\end{tabular}

$1=$ Ace poder limón ${ }^{\circledR}$

$2=$ Ariel Limón ${ }^{\circledR}$

L.C. = límite de confianza;

$\mathrm{F}=$ estadístico de Fisher;

$\mathrm{P}=$ Probabilidad;

NOEC $=$ concentración de efectos no observables;

LOEC $=$ concentración más baja de efectos

observables.

Letras iguales minúsculas en una misma línea vertical indican porcentaje de mortalidad estadísticamente iguales, según los resultados de la prueba de Student-Newman-Keuls (S-N-K). (SPSS versión 7,5)

Letras iguales mayúsculas en una misma línea horizontal indican porcentaje de mortalidad estadísticamente iguales según los resultados de la prueba de Student-Newman-Keuls (S-N-K). (SPSS versión 7,5) 
Tabla 3. Concentración Letal media $\left(\mathrm{CL}_{50}\right)$, NOEC, LOEC de Heleobia cumingii expuesta al detergente alquil aril sulfonato de sodio (LAS) por $48 \mathrm{~h}$.

\begin{tabular}{|c|c|c|}
\hline $\begin{array}{c}\text { Concentración } \\
\mathrm{mg} \mathrm{L}^{-1}\end{array}$ & $\begin{array}{c}\% \text { Mortalidad } \\
1 \\
\end{array}$ & $\begin{array}{c}\% \text { Mortalidad } \\
2 \\
\end{array}$ \\
\hline 0 & $0 \mathrm{aA}$ & $0 \mathrm{aA}$ \\
\hline 28,12 & $5 \mathrm{aA}$ & $10 \mathrm{aA}$ \\
\hline 56,25 & $50 \mathrm{bA}$ & $35 \mathrm{abA}$ \\
\hline 112,5 & $65 \mathrm{bcA}$ & $60 \mathrm{bcA}$ \\
\hline 225 & $85 \mathrm{bcA}$ & $85 \mathrm{bcA}$ \\
\hline 450 & $95 \mathrm{cA}$ & $95 \mathrm{cA}$ \\
\hline $\begin{array}{l}\mathrm{CL}_{50}\left(\mathrm{mg} \mathrm{L}^{-1}\right) \\
\text { L.C. inferior }(\mathrm{mg}\end{array}$ & 78,80 & 87,07 \\
\hline $\begin{array}{l}\left.\mathrm{L}^{-1}\right) \\
\text { L.C. superior } \\
\left(\mathrm{mg} \mathrm{L}^{-1}\right)\end{array}$ & $\begin{array}{c}57,11 \\
104,81\end{array}$ & $\begin{array}{c}63,76 \\
115,92\end{array}$ \\
\hline$F_{\text {concentraciones }}$ & 23,44 & 19,99 \\
\hline $\mathrm{P}$ & 0,00 & 0,001 \\
\hline $\mathrm{F}_{\text {repeticiones }}$ & 1,25 & 0,97 \\
\hline $\mathrm{P}$ & 0,33 & 0,55 \\
\hline $\operatorname{NOEC}\left(\mathrm{mg} \mathrm{L}^{-1}\right)$ & 56,25 & 112,5 \\
\hline $\operatorname{LOEC}\left(\mathrm{mg} \mathrm{L}^{-1}\right)$ & 112,5 & 225 \\
\hline
\end{tabular}

$1=$ Ace poder limón $\mathbb{R}$

$2=$ Ariel Limón ${ }^{\circledR}$

L.C.= límite de confianza;

$\mathrm{F}=$ estadístico de Fisher;

$\mathrm{P}=$ Probabilidad;

NOEC $=$ concentración de efectos no observables;

LOEC $=$ concentración más baja de efectos

observables.

Letras iguales minúsculas en una misma línea vertical indican porcentaje de mortalidad estadísticamente iguales, según los resultados de la prueba de Student-Newman-Keuls (S-N-K). (SPSS versión 7,5).

Letras iguales mayúsculas en una misma línea horizontal indican porcentaje de mortalidad estadísticamente iguales según los resultados de la prueba de Student-Newman-Keuls (S-N-K). (SPSS versión 7,5).
Tabla 4. Respuestas de Toxicidad de diversos bioensayos expuestos al surfactante aniónico alquil aril sulfonato de sodio (LAS).

\begin{tabular}{|c|c|c|}
\hline Organismos biológicos & $\begin{array}{l}\mathrm{CL}_{50} / \mathrm{EC}_{50} \\
\left(\mathrm{mg} \mathrm{IA} \mathrm{L}^{-1}\right)\end{array}$ & Referencias \\
\hline $\begin{array}{l}\text { Partículas } \\
\text { submitocondriales de } \\
\text { bovinos* }(12 \mathrm{~h})\end{array}$ & 0,6 & $\begin{array}{l}\text { Argese et al. } \\
(1997)\end{array}$ \\
\hline $\begin{array}{l}\text { Lepomis macrochirus } \\
\text { (Piscis) }(96 \mathrm{~h})\end{array}$ & 3 & $\begin{array}{l}\text { Argese } \text { et al. } \\
\text { (1997) }\end{array}$ \\
\hline $\begin{array}{l}\text { Daphnia magna } \\
\text { (Invertebrado) (48 h) }\end{array}$ & 2,3 & $\begin{array}{l}\text { Argese et al. } \\
\text { (1997) }\end{array}$ \\
\hline $\begin{array}{l}\text { Daphnia magna } \\
\text { (Invertebrado) }(48 \mathrm{~h})\end{array}$ & $4-85$ & $\begin{array}{l}\text { Pettersson et } \\
\text { al. }(2000)\end{array}$ \\
\hline $\begin{array}{l}\text { Ceriodaphnia dubia } \\
\text { (Invertebrado) }(48 \mathrm{~h})\end{array}$ & 1,50 & $\begin{array}{l}\text { Morgan \& } \\
\text { Oude (1993) }\end{array}$ \\
\hline $\begin{array}{l}\text { Selenastrum } \\
\text { capricornutum } \\
\text { (microalga) }(96 \mathrm{~h})\end{array}$ & 29 & $\begin{array}{l}\text { Argese et al. } \\
\text { (1997) }\end{array}$ \\
\hline $\begin{array}{l}\text { Microcystis sp. } \\
\text { (microalga) }(96 \mathrm{~h})\end{array}$ & 0,09 & Lewis (1986) \\
\hline $\begin{array}{l}\text { Melanoides tuberculata } \\
\text { (Mollusca) }(48 \mathrm{~h})\end{array}$ & 201,97 & Este estudio \\
\hline $\begin{array}{l}\text { Physa venustula } \\
\text { (Mollusca) (48 h) }\end{array}$ & 71,41 & Este estudio \\
\hline $\begin{array}{l}\text { Heleobia cumingii } \\
\text { (Mollusca) }(48 \mathrm{~h})\end{array}$ & 82,93 & Este estudio \\
\hline
\end{tabular}

